\title{
Effect of star architecture \\ on the dynamics of 1,4-cis-polyisoprene under nanometer confinement
}

Panagiotis Kardasis, ${ }^{1}$ Angelos Oikonomopoulos, ${ }^{2}$ Georgios Sakellariou, ${ }^{2}$ Martin Steinhart, ${ }^{3}$ and George Floudas ${ }^{1,4^{*}}$

${ }^{1}$ Department of Physics, University of Ioannina, 45110 Ioannina, Greece

${ }^{2}$ Department of Chemistry, National and Kapodistrian University of Athens, 15771 Athens, Greece

${ }^{3}$ Institut für Chemie neuer Materialien, Universität Osnabrück, D-49069 Osnabrück, Germany

${ }^{4}$ University Research Center of Ioannina (URCI) - Institute of Materials Science and Computing, 45110 Ioannina, Greece 


\section{Synthesis}

Materials: $n$-Butyllithium (1.6 M in hexane), calcium hydride $\left(\mathrm{CaH}_{2}\right)$, were purchased from Aldrich and were used as received. Benzene (Acros), isoprene (Aldrich) were purified according to the standards of anionic polymerization high vacuum techniques as described elsewhere. ${ }^{1,2}$ sec-BuLi ( $n$ hexane solution) was prepared in vacuum by the reaction of excess lithium metal with sec-butyl chloride (Acros). Sec-butyl chloride was freeze-degassed three times on the vacuum line, fractionally distilled into calibrated ampoules, and stored at room temperature. Methanol (Acros) was degassed several times and distilled in ampules.

Synthesis of "living" polyisoprene: "Living" polyisoprene, $\mathrm{PI}^{-} \mathrm{Li}^{+}$, was synthesized in benzene using standard all-glass high-vacuum techniques. The polymerization was performed in a reactor shown in Figure S1. The reactor was connected to a purge section containing a $250 \mathrm{~mL}$ flask with a septum inlet. The ampules containing initiator, and monomer were connected to the reactor, and the entire reactor was evacuated to attain high vacuum. Then, a small amount of $n$-BuLi (1.6 M in hexane, $5 \mathrm{~mL}$ ) was added into the reactor under static vacuum through a septum using a syringe, and the septum portion was removed by sealing the glass constriction, which was thoroughly washed by distilling hexane above the portion of the constriction using a wet liquid nitrogen towel. The hexane solution of $n$-BuLi was frozen and degassed thoroughly under high vacuum. $100 \mathrm{~mL}$ of benzene was distilled into the purge flask under high vacuum, and the entire reactor was sealed off from the vacuum line. The benzene containing $n$-BuLi was rinsed throughout the reactor to quench any impurity present on the glass surface. The inner walls of the reactor were washed thoroughly by condensing pure benzene. Then, the reactor was positioned in a way that the pure benzene could be distilled into the reactor. The purge section containing the washed $n$-BuLi was removed from the reactor through heatsealing of the glass constriction. Subsequently, the break-seals of the ampules containing isoprene $(5.0 \mathrm{~g}, 0.073 \mathrm{~mol})$ and $s$-BuLi $\left(10^{-3} \mathrm{~mol}\right)$ were opened and mixed with benzene. The polymerization was allowed to proceed for $24 \mathrm{~h}$ at $25{ }^{\circ} \mathrm{C}$. After the completion of isoprene polymerization, a small amount of butadiene was added in order to end-capped the polymer chains with 1-2 monomeric units of butadiene. An aliquot of the living polymer was collected in an ampule and terminated with degassed methanol for further characterization. Finally, the solution with the living polyisoprene was collected in a round-bottom flask equipped with break-seal and removed by the reactor by heatsealing the constriction. 
Synthesis of Polyisoprene Stars: Polyisoprene homopolymer stars (PI) $)_{\mathrm{n}}$, having different functionalities, $n,(n=6,8$, and 64 number of arms) and different arm molecular weights (Table 1) were synthesized in benzene using standard all-glass high-vacuum techniques. The linking reaction between the "living" polyisoprene and the appropriate chlorosilane $\left[(\mathrm{Si}-\mathrm{Cl})_{6},(\mathrm{Si}-\mathrm{Cl})_{8}\right.$, and $\left.(\mathrm{Si}-\mathrm{Cl})_{64}\right]$ was performed in a reactor shown in Figure S2 and the procedure followed was similar to the one described above. ${ }^{3}$ The linking reaction took place in benzene, at concentration $10 \% \mathrm{w} / \mathrm{v}$ of the total polymer weight. The apparatus was rinsed as described above, living polyisoprene lithium was added, followed by the appropriate chlorosilane. An excess of $15 \%$ mol of the "living" polyisoprene in respect to the $\mathrm{Si}-\mathrm{Cl}$ mol was used. After the completion of the linking reaction the excess of the linear polyisoprene homopolymer was removed by fractionation in a toluene/methanol solvent/non-solvent system. Size exclusion chromatography (SEC), nuclear magnetic resonance (NMR) spectroscopy, and static light scattering (SLS), were performed in order to fully characterize the synthesized star polymers.

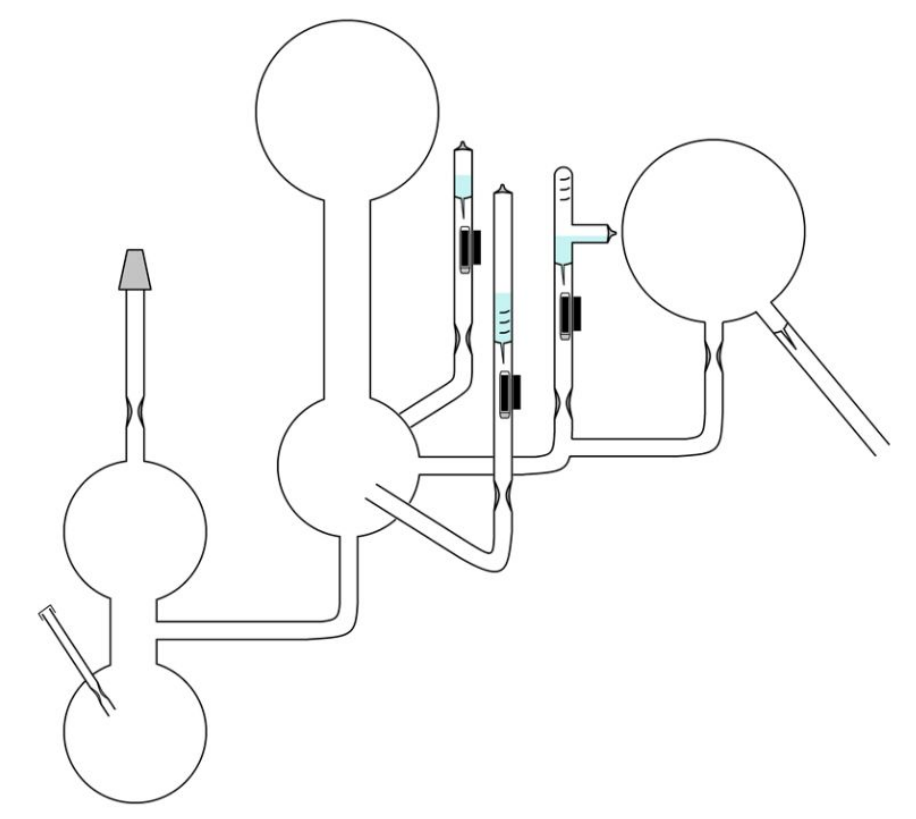

Figure S1. Polymerization apparatus for the synthesis of "living" polyisoprene. 


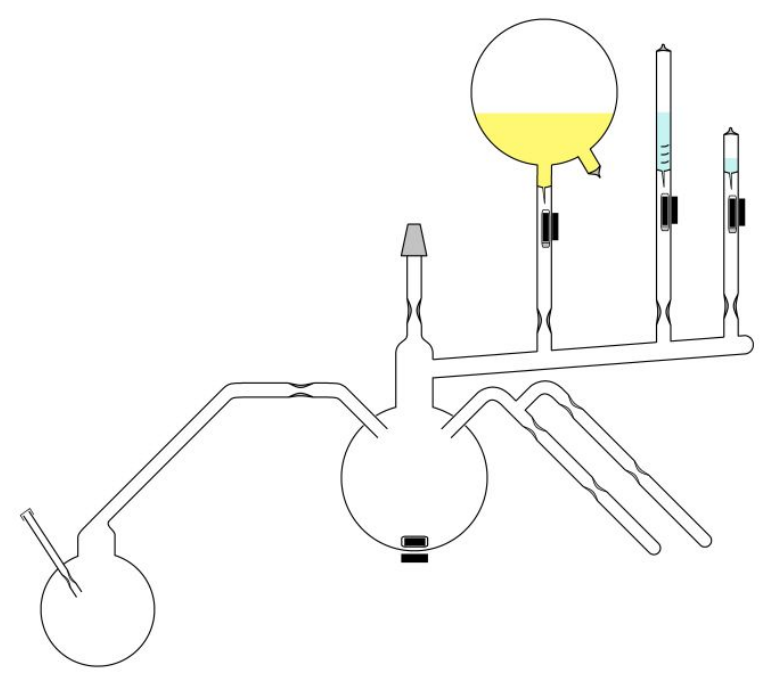

Figure S2. Glass apparatus for the synthesis of polyisoprene stars.

PI 64 ARMS 1H-NMR

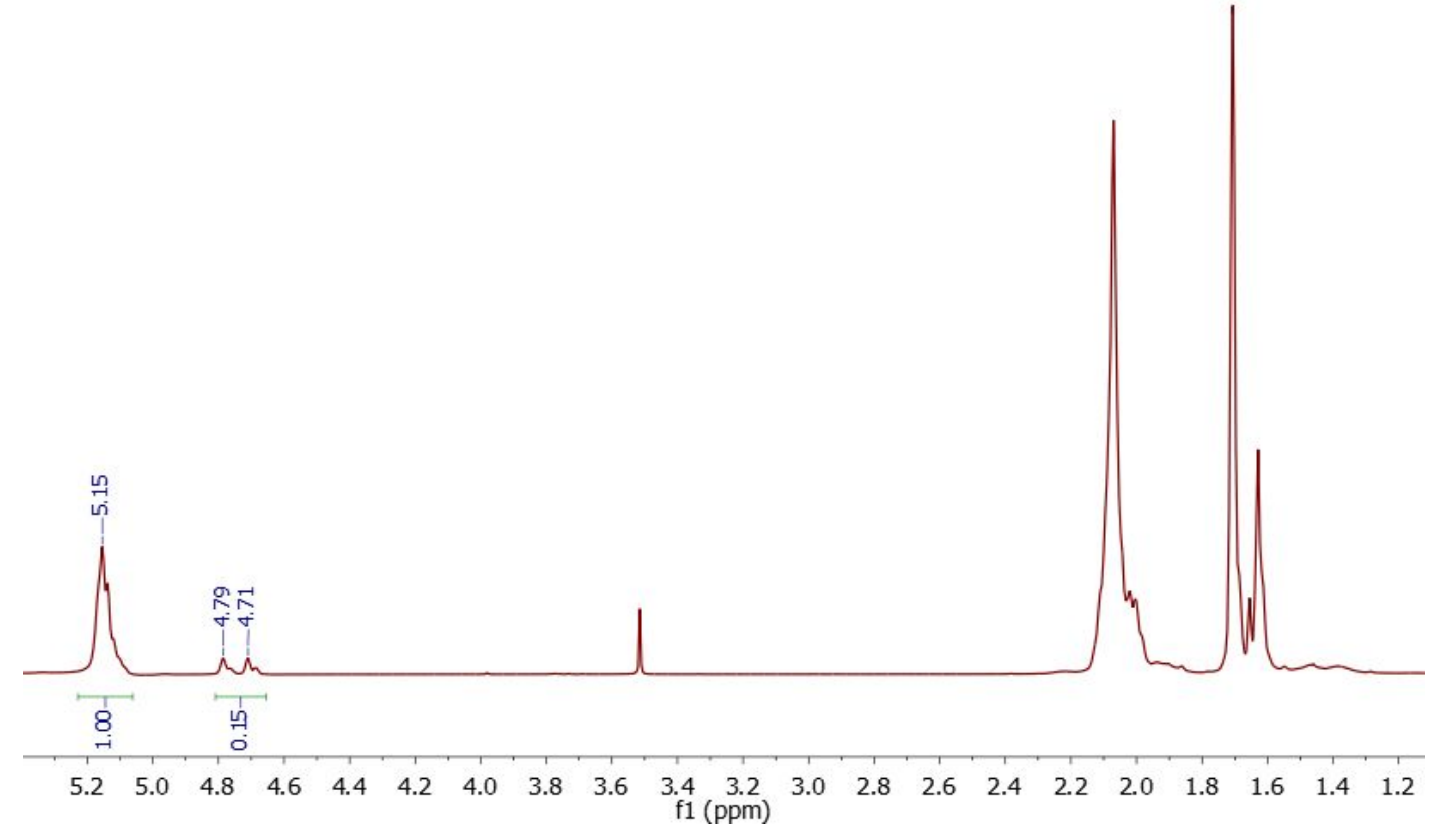

Figure S3. ${ }^{1} \mathrm{H}-\mathrm{NMR}$ spectrum of SPI-64-5 in $\mathrm{CDCl}_{3}$. 


\section{${ }^{13} \mathrm{C}-\mathrm{NMR}$}

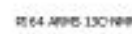

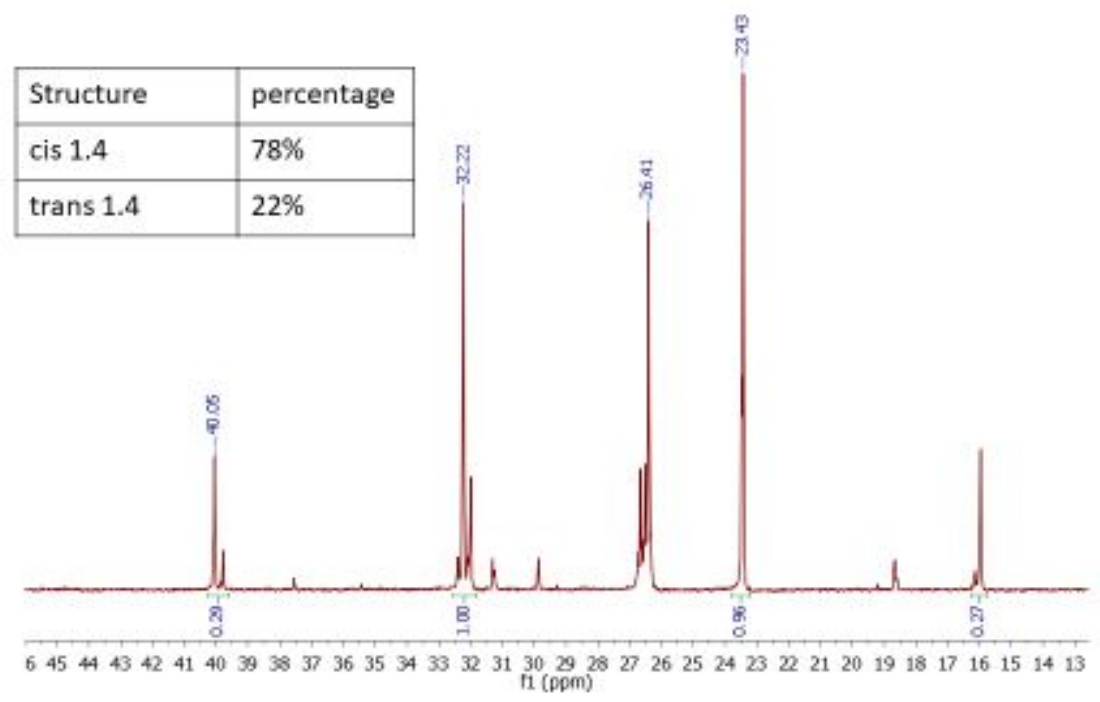

Figure S4. ${ }^{13} \mathrm{C}-\mathrm{NMR}$ spectrum of SPI-64-5in $\mathrm{CDCl}_{3}$.

— PI 6 ARMS 13.5K

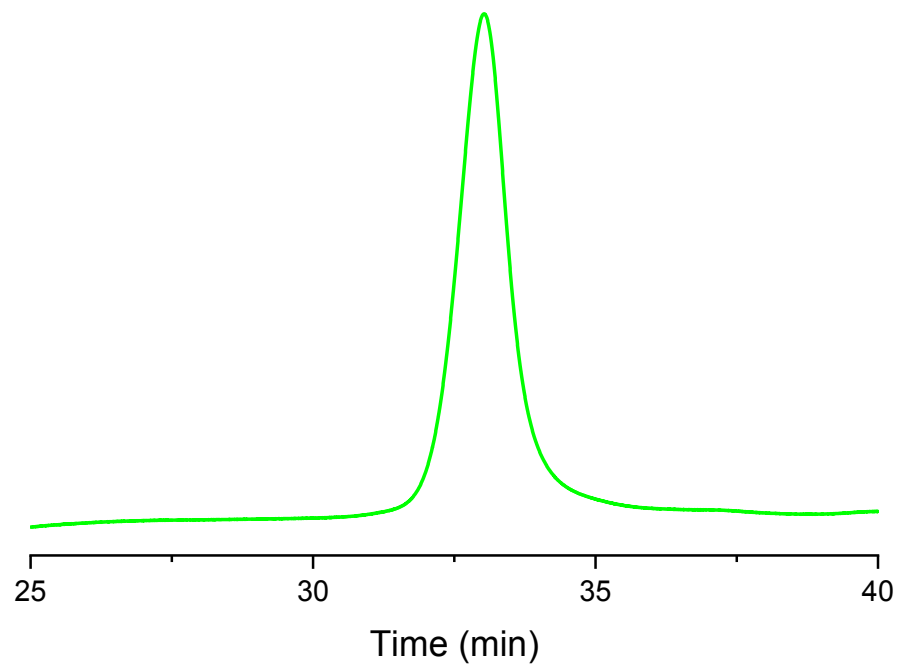

Figure S5. SEC chromatogram of SPI-6-13.5 in $\mathrm{CHCl}_{3}$ at $25^{\circ} \mathrm{C}$. 


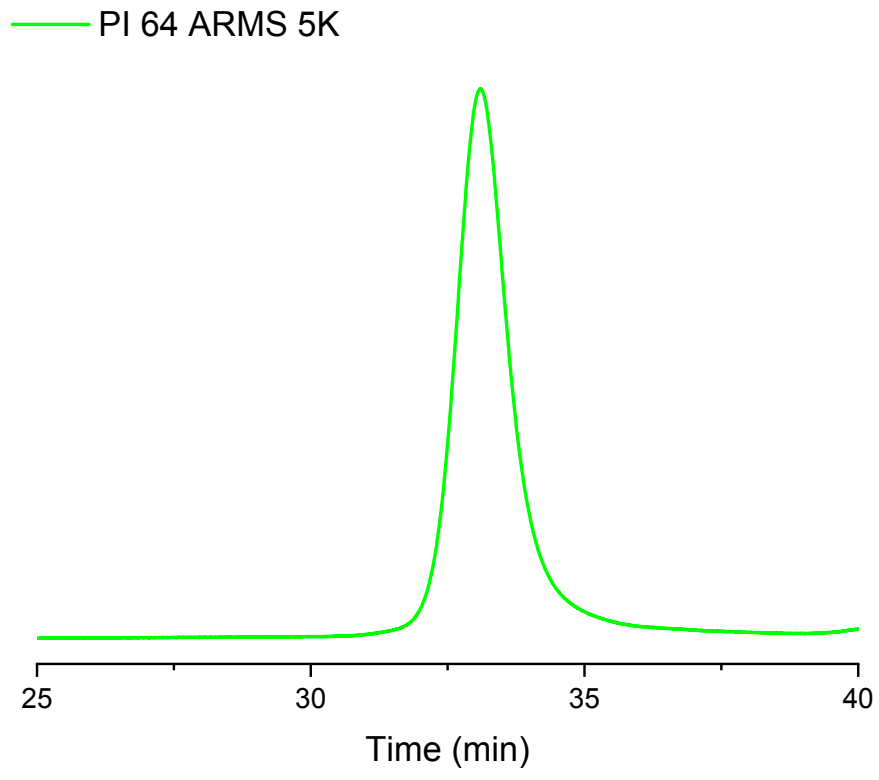

Figure S6. SEC chromatogram of SPI-64-5 in $\mathrm{CHCl}_{3}$ at $25^{\circ} \mathrm{C}$.

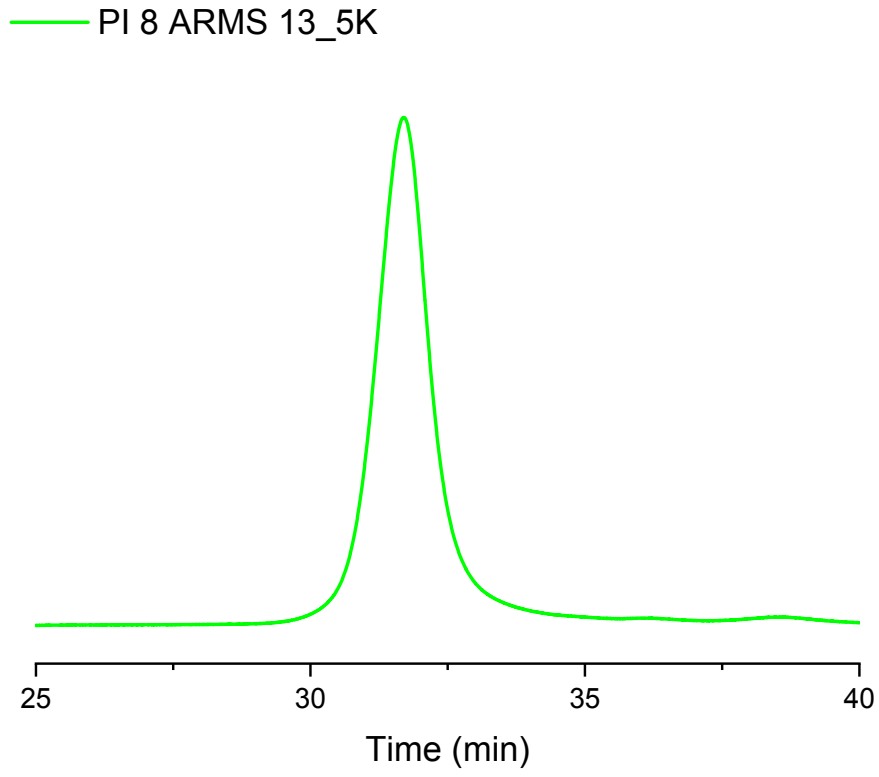

Figure S7. SEC chromatogram of SPI-8-13.5 in $\mathrm{CHCl}_{3}$ at $25^{\circ} \mathrm{C}$. 


\section{Rheology}
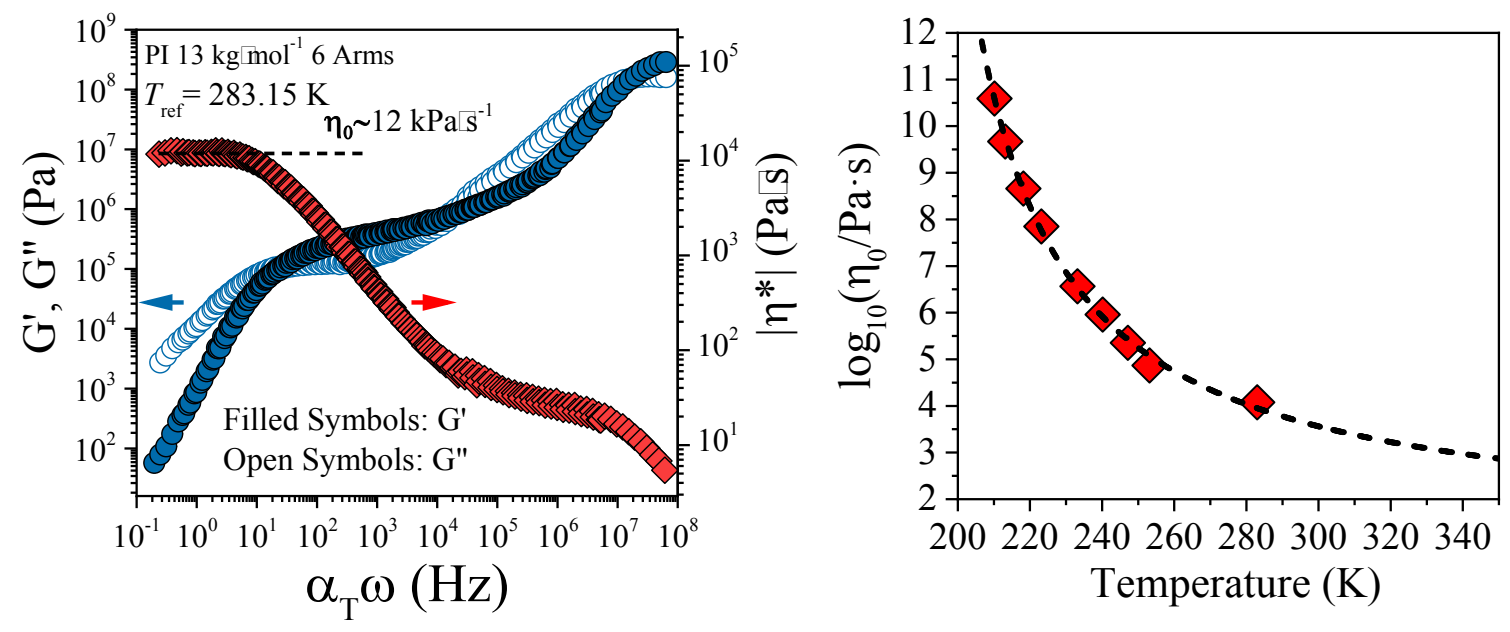

Figure S8. (left) Master curve of the frequency-dependent storage ( $G^{\prime}$, filled symbols) and loss $\left(G^{\prime \prime}\right.$, open symbols) moduli of SPI-6-13.5 at $T_{\text {ref }}=283.15 \mathrm{~K}$. The viscosity is shown by the red rhombi. The value of the zero-shear viscosity at the $T_{\text {ref }}$ is shown by the horizontal line. (right) The zero viscosity as function of the temperature. The curved dash line represents fit with the VFT equation.

\section{Dielectric Spectroscopy}

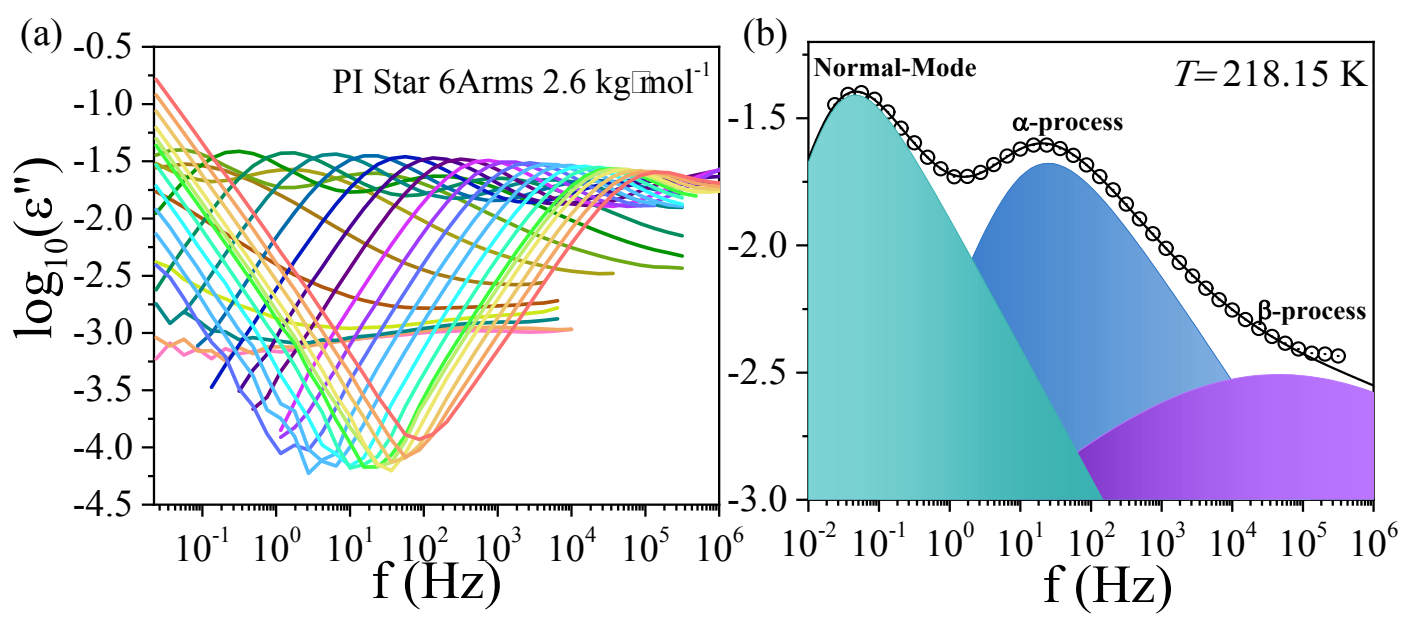

Figure S9. (a) Dielectric loss curves of SPI-6-2.6 in the bulk in range from $183.15 \mathrm{~K}$ to $313.15 \mathrm{~K}$. (b) The dielectric loss curve at selected temperature $T=218.15 \mathrm{~K}$. The black line represents a fit with a summation of three Havriliak-Negami functions for: the slower Normal-Mode (light blue), the intermediate $\alpha$-process (blue) and the faster $\beta$-process (purple). 


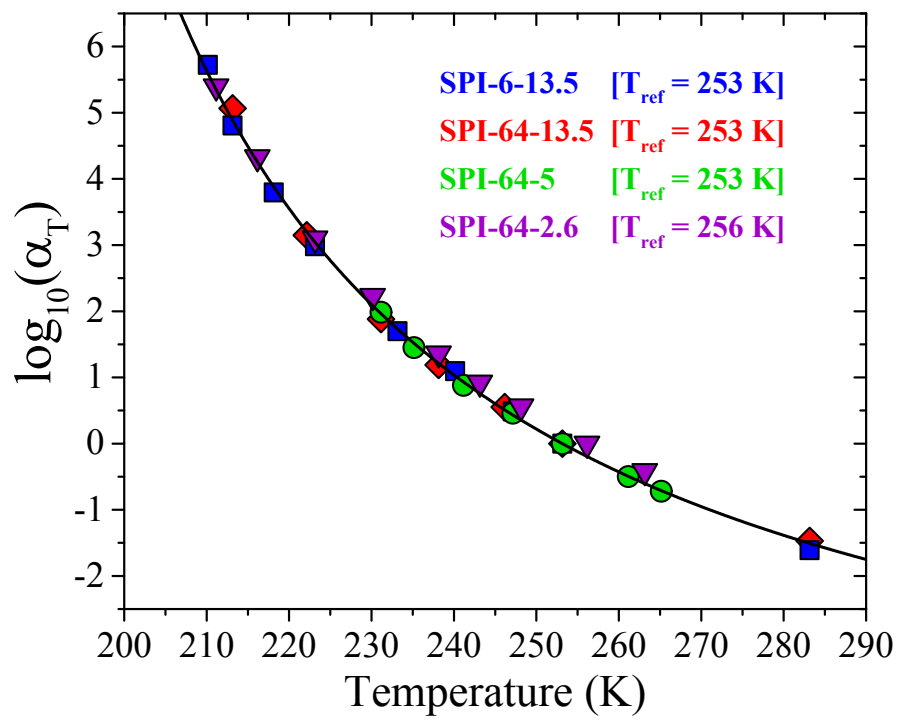

Figure S10. Horizontal shift factors, $\alpha_{\mathrm{T}}$, of SPI-6-13.5 (blue), SPI-64-13.5 (red) and SPI-64-5 (green) at $T_{\text {ref }}=253 \mathrm{~K}$ and SPI-64-2.6 (purple) at $T_{\text {ref }}=256 \mathrm{~K}$ as a function of temperature.
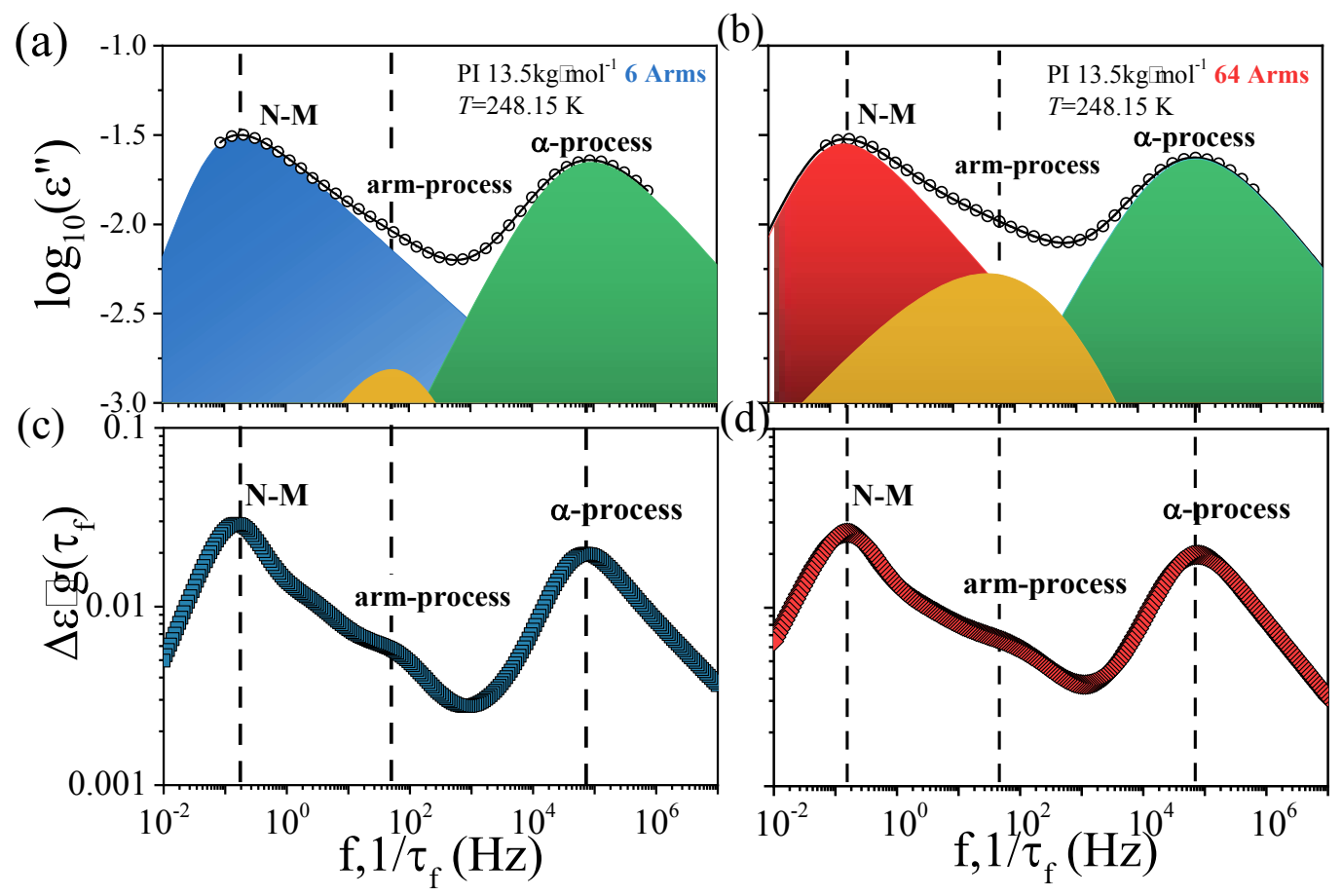
Figure S11. Dielectric loss curves of (a) SPI-6-13.5 and (b) SPI-64-13.5 as function of frequency at selected temperature of $248.15 \mathrm{~K}$. The black line represents fit with a summation of three HavriliakNegami functions for: $\alpha$-process (green), arm-process (orange) and $N M$ (blue for $f=6$ and red for $f=$ 64). (c), (d) The distribution of relaxation times as function of $1 / \tau_{\mathrm{f}}$ where $\tau_{\mathrm{f}}=1 / \mathrm{f}_{\max }$. The vertical dash lines indicate the characteristic (maximum) frequency of each process.

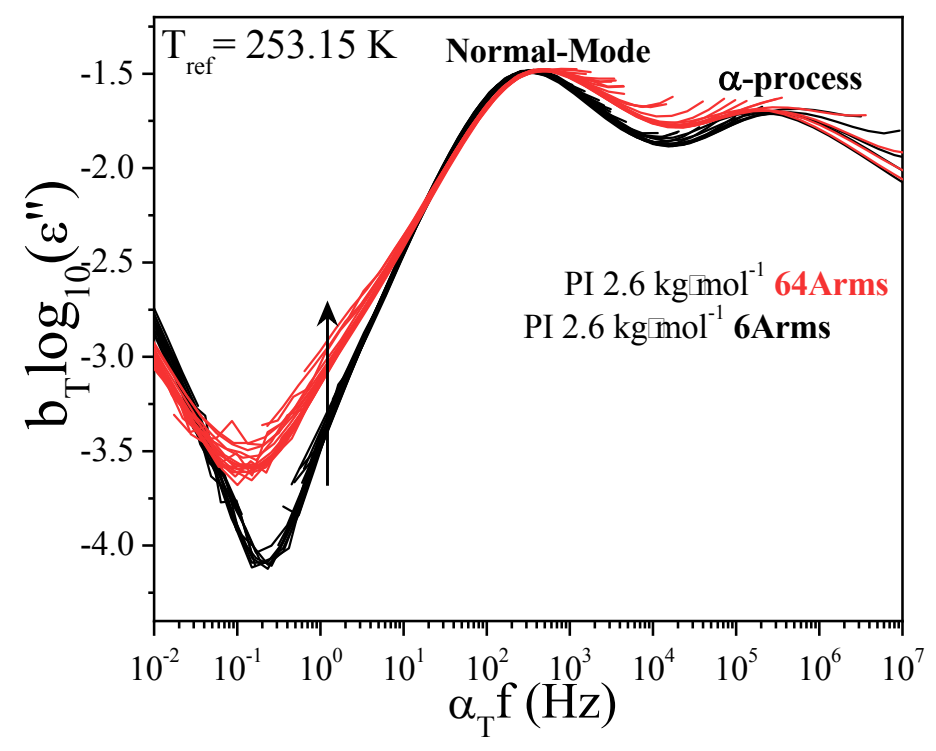

Figure S12. Comparison of the superimposed dielectric loss curves of SPI-6-2.6 (black) and SPI-642.6 (red) as a function of frequency at $T_{\text {ref }}=253.15 \mathrm{~K}$. At lower frequencies the broadening of the curves with increasing $f$ is clearly evident (indicated by the arrow). 

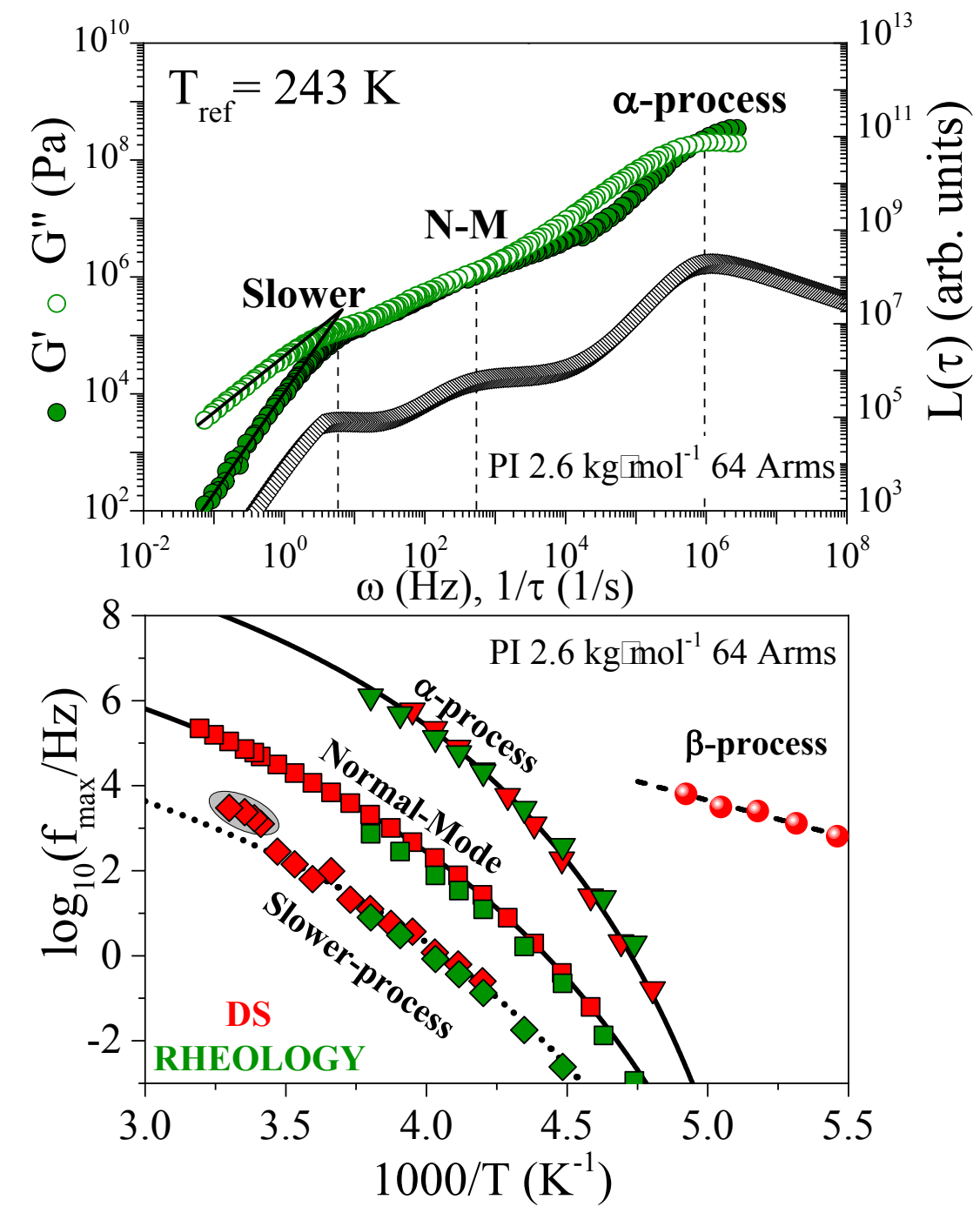

Figure S13. (Top) Master curves of the frequency-dependent storage ( $G^{\prime}$, filled green symbols) and loss ( $G^{\prime \prime}$, open green symbols) moduli of SPI-64-2.6 at $T_{\text {ref }}=243.15 \mathrm{~K}$. The black symbols correspond to the distribution of relaxation times, $L(\tau)$, plotted as a function of $1 / \tau$. (Bottom) The activation plot of the characteristic frequencies for SPI-64-2.6; $\beta$-process (spheres), segmental $\alpha$-process (down triangles), normal-mode (squares) and the slower (soft-colloidal) process (rhombi) obtained from rheology (green) and DS (red). 


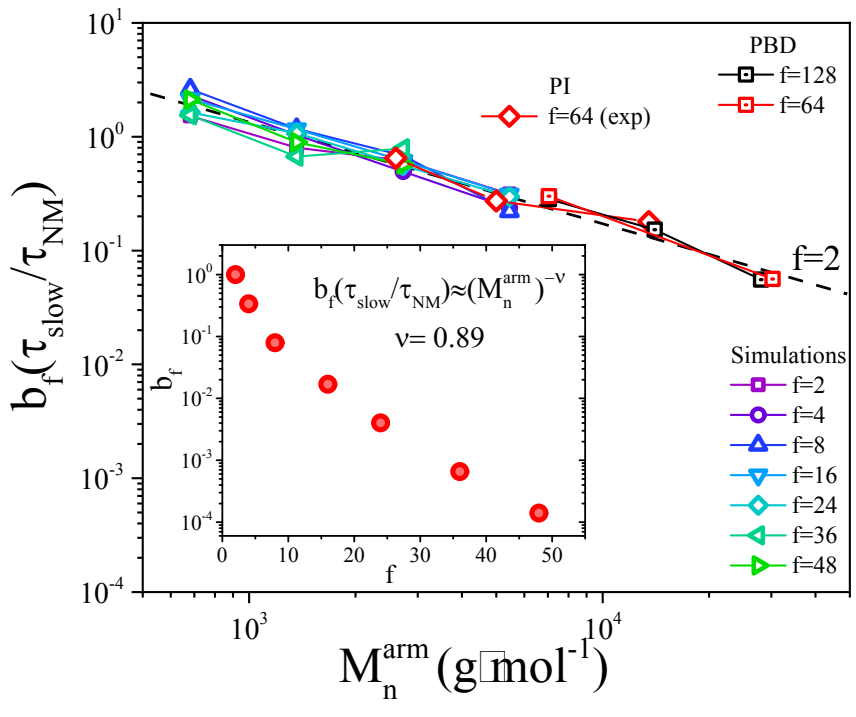

Figure S14. The relaxation times of the slower (soft-colloidal) process normalized by the corresponding times for the $N M$ of the star-shaped PI with $f=64$ (red rhombi) as function of molecular mass of the arms. The simulated data and the relaxations times for the PBD were obtained from. ${ }^{4}$ The data were shifted to the values of $f=2$ and follow a linear trend with a slope of -0.89 . Then inset gives the shift factor for the simulated data.

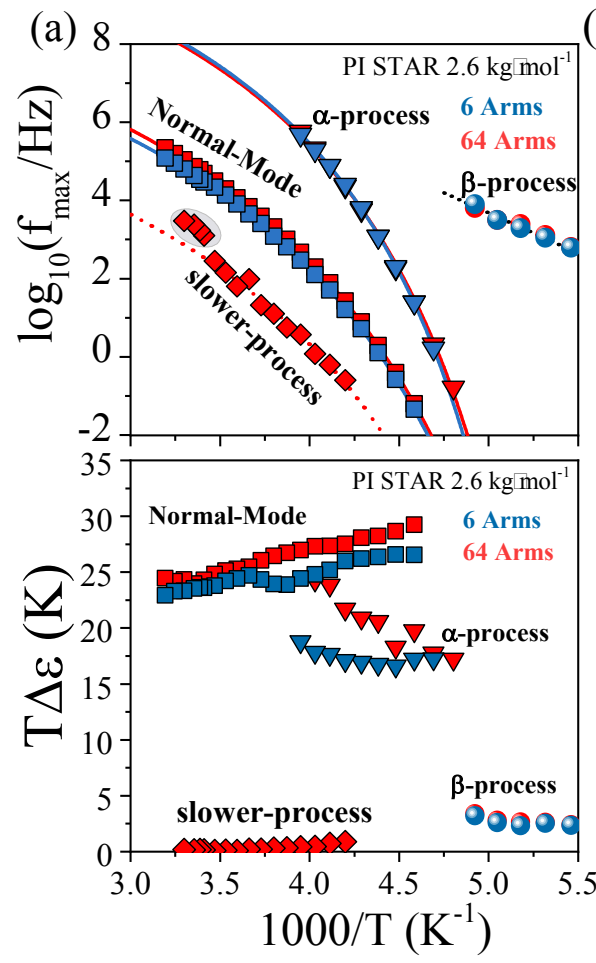

(b)

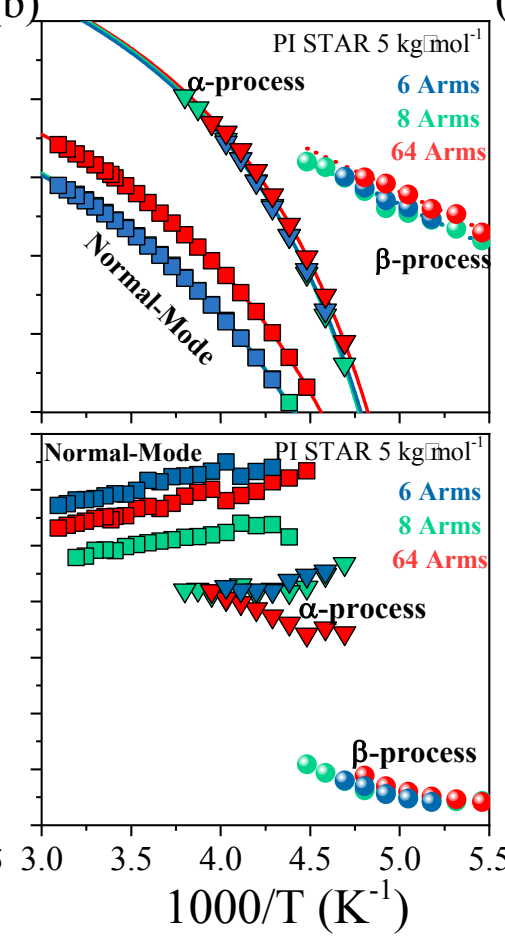

(c)

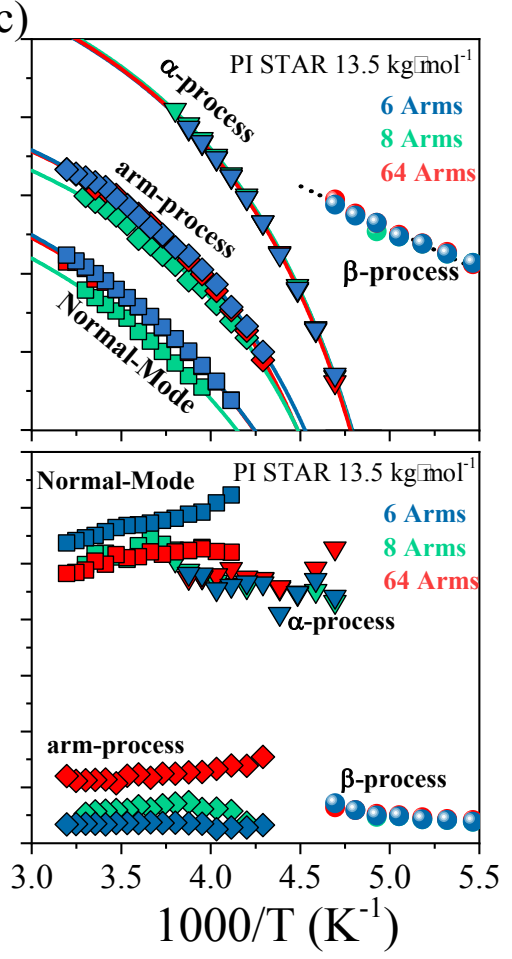

Figure S15. (top) Activation plot of the characteristic frequencies for the star-shaped PI with molar mass of (a) $M_{n}=2.6 \mathrm{~kg} \cdot \mathrm{mol}^{-1}$, (b) $M_{n}=5 \mathrm{~kg} \cdot \mathrm{mol}^{-1}$ and (c) $M_{n}=13.5 \mathrm{~kg} \cdot \mathrm{mol}^{-1}$ with 6 arms (blue), 8 arms (green) and 64 arms (red) for the $\beta$-process (spheres), the segmental $\alpha$-process (down triangles), the normal-mode (squares), the slow (soft-colloidal) process (rhombi, unentangled PI) and the arm- 
process (rhombi, entangled PI). The curved and the straight lines represent fits with the VFT and Arrhenius equations, respectively. (bottom) Dielectric strength multiplied with temperature and plotted as a function of the inverse temperature 1000/T (each process is shown with the corresponding color and symbol).

Table S1. Fit Parameters of the Characteristic Frequencies of the Star-Shaped PI $\left(M_{n}=13.5\right.$ $\mathrm{kg} \cdot \mathrm{mol}^{-1}$ ) with the VFT and Arrhenius equation.

\begin{tabular}{|c|ccc|ccc|ccc|cc|c|}
\hline $\begin{array}{c}\text { STAR PI } \\
\begin{array}{c}13.5 \\
\mathrm{~kg} \cdot \mathrm{mol}^{-1}\end{array}\end{array}$ & \multicolumn{3}{|c}{ Normal-Mode } & \multicolumn{3}{c|}{$\alpha$-process } & \multicolumn{3}{c|}{ arm-process } & \multicolumn{2}{c|}{$\beta$-process } & $\left(\begin{array}{c}\left(T_{\mathrm{g}} \pm 1\right) \\
(\mathrm{K})\end{array}\right.$ \\
\hline $6 \mathrm{Arms}$ & $6.9 \pm 0.1$ & $1603 \pm 48$ & $158 \pm 2$ & $12^{\#}$ & $1287 \pm 9$ & $169 \pm 1$ & $8.3 \pm 0.2$ & $1175 \pm 103$ & $172 \pm 3$ & 14 & $41 \pm 1$ & $207 \pm 1$ \\
\hline $8 \mathrm{Arms}$ & $6.4 \pm 0.1$ & $1603 \pm 69$ & $158 \pm 1$ & $12^{\#}$ & $1250 \pm 8$ & $170 \pm 1$ & $7.7 \pm 0.1$ & $1136 \pm 26$ & $172 \pm 3$ & 14 & $42 \pm 2$ & $207 \pm 1$ \\
\hline $64 \mathrm{Arms}$ & $7.0 \pm 0.5$ & $1640 \pm 89$ & $152 \pm 8$ & $12^{\#}$ & $1271 \pm 5$ & $170 \pm 1$ & $8.9 \pm 0.5$ & $1281 \pm 206$ & $170 \pm 6$ & 14 & $42 \pm 1$ & $207 \pm 1$ \\
\hline
\end{tabular}

${ }^{\#}$ Held constant

Table S2. Fit Parameters of Star-Shaped PI $\left(M_{\mathrm{n}}=5 \mathrm{~kg} \cdot \mathrm{mol}^{-1}\right)$ with the VFT and Arrhenius equation.

\begin{tabular}{|c|c|c|c|c|c|c|c|c|c|}
\hline \multirow{2}{*}{$\begin{array}{c}\text { STAR PI } \\
5 \mathrm{~kg} \cdot \mathrm{mol}^{-1}\end{array}$} & \multicolumn{3}{|c|}{ Normal-Mode } & \multicolumn{3}{|c|}{$\alpha$-process } & \multicolumn{2}{|c|}{$\beta$-process } & \multirow{2}{*}{$\begin{array}{c}\left(T_{\mathrm{g}} \pm 1\right) \\
(\mathrm{K})\end{array}$} \\
\hline & $\log \left(f_{\infty}\right)$ & $B$ & $T_{0}(\mathrm{~K})$ & $\log \left(f_{\infty}\right)$ & $B$ & $T_{0}$ & $\log \left(f_{\infty}\right)$ & $E\left(\mathrm{~kJ} \cdot \mathrm{mol}^{-1}\right)$ & \\
\hline $6 \mathrm{~A}$ & $8.0 \pm 0.1$ & 511 & & $12^{\#}$ & $0=$ & & 14 & & $206 \pm 1$ \\
\hline $8 A$ & \pm 0.1 & $1724=$ & 1 & $12^{\#}$ & 1259 & $1^{\prime}$ & 14 & $41 \pm 1$ & $206 \pm 1$ \\
\hline 64 Arms & $8.9 \pm 0.1$ & $1550 \pm 27$ & $158 \pm 1$ & $12^{\#}$ & $1275 \pm 140$ & $169 \pm 3$ & 14 & $43 \pm 2$ & $205 \pm 1$ \\
\hline
\end{tabular}

${ }^{\#}$ Held constant

Table S3. Fit Parameters of Star-Shaped PI $\left(M_{\mathrm{n}}=2.6 \mathrm{~kg} \cdot \mathrm{mol}^{-1}\right)$ with the VFT and Arrhenius equation.

\begin{tabular}{|c|c|c|c|c|c|c|c|c|c|c|c|}
\hline \multirow{2}{*}{$\begin{array}{c}\text { STAR } \\
\text { PI } 2.6 \\
\mathrm{~kg} \cdot \mathrm{mol}^{-} \\
1\end{array}$} & \multicolumn{2}{|c|}{ Normal-Mode } & \multicolumn{3}{|c|}{$\alpha$-process } & \multicolumn{3}{|c|}{ slower-process } & \multicolumn{2}{|c|}{$\beta$-process } & \multirow{2}{*}{$\begin{array}{c}\left(T_{\mathrm{g}} \pm 1\right) \\
(\mathrm{K})\end{array}$} \\
\hline & $\log \left(f_{\infty}\right)$ & $T_{0}(\mathrm{~K})$ & $\log \left(f_{\infty}\right)$ & $B$ & $T_{0}(\mathrm{~K})$ & $\log \left(f_{\infty}\right)$ & $B$ & $T_{0}(\mathrm{~K})$ & $\log \left(f_{\infty}\right)$ & $E\left(\mathrm{~kJ} \cdot \mathrm{mol}^{-1}\right)$ & \\
\hline 6 Arms & $9.4 \pm 0.1 \quad 1548 \pm 30$ & $156 \pm 1$ & $12^{\#}$ & $1247 \pm 49$ & $167 \pm 2$ & -- & -- & -- & 14 & $40 \pm 2$ & $204 \pm 1$ \\
\hline 64 Arms & $9.6 \pm 0.1 \quad 1591 \pm 45$ & $155 \pm 1$ & $12^{\#}$ & $1292 \pm 30$ & $165 \pm 1$ & $7.5 \pm 0.2$ & 1591 & $153 \pm 2$ & 14 & $40 \pm 2$ & $203 \pm 1$ \\
\hline
\end{tabular}

${ }^{\#}$ Held constant 


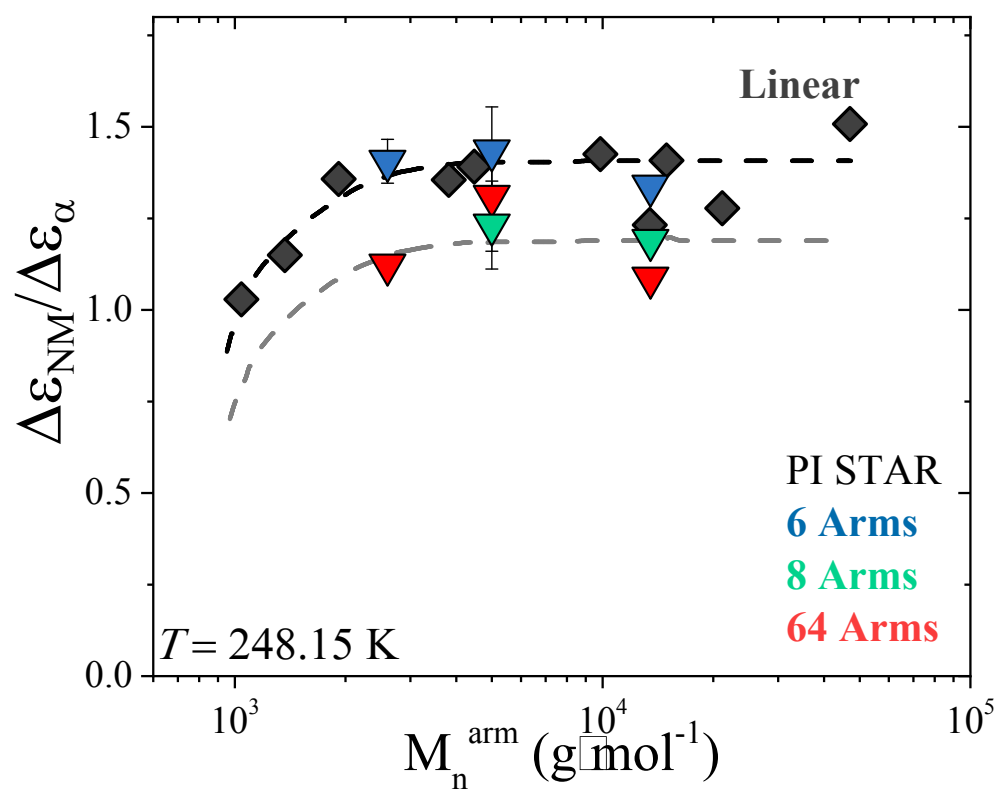

Figure S16. The dielectric strength of the normal mode normalized by the corresponding segmental strength of linear polyisoprene (black rhombi) ${ }^{5}$ and star-shaped (down triangles) with: $f=6$ (blue), $f$ $=8$ (green) and $f=64$ (red) as a function of the molar mass of the arm at $T=248.15 \mathrm{~K}$

\section{Star Polyisoprenes in Confinement}

(a)

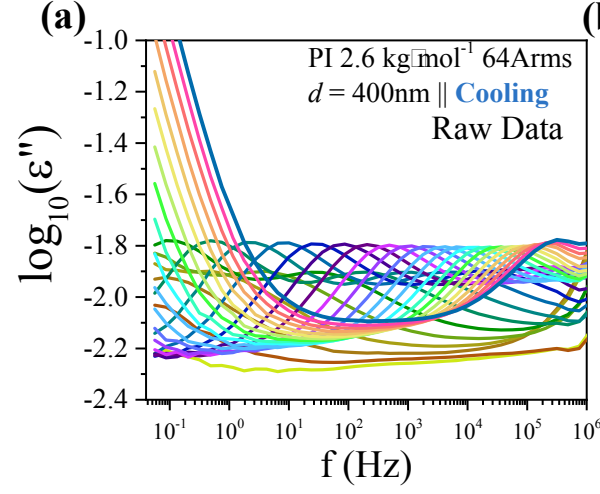

(b)

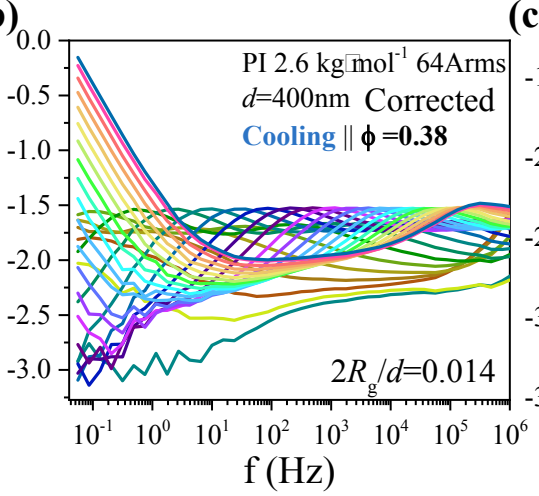

(c)

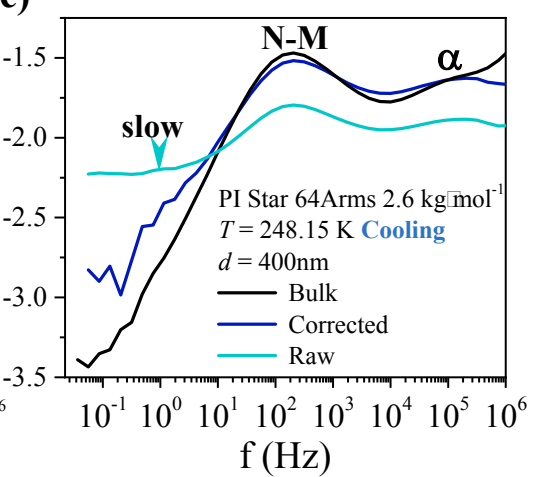

Figure S17. (a) Dielectric loss curves of star-shaped SPI-64-2.6 under confinement in AAO templates with a diameter of $400 \mathrm{~nm}$ as a function of frequency in the temperature range of $178.15 \mathrm{~K}$ to 323.15 K. (b) The corrected dielectric loss curves with porosity of 0.38 . (c) The bulk (black), the raw/measured (light blue) under confinement and the corrected (blue) dielectric loss curves as function of frequency at selected temperature of $248 \mathrm{~K}$. 

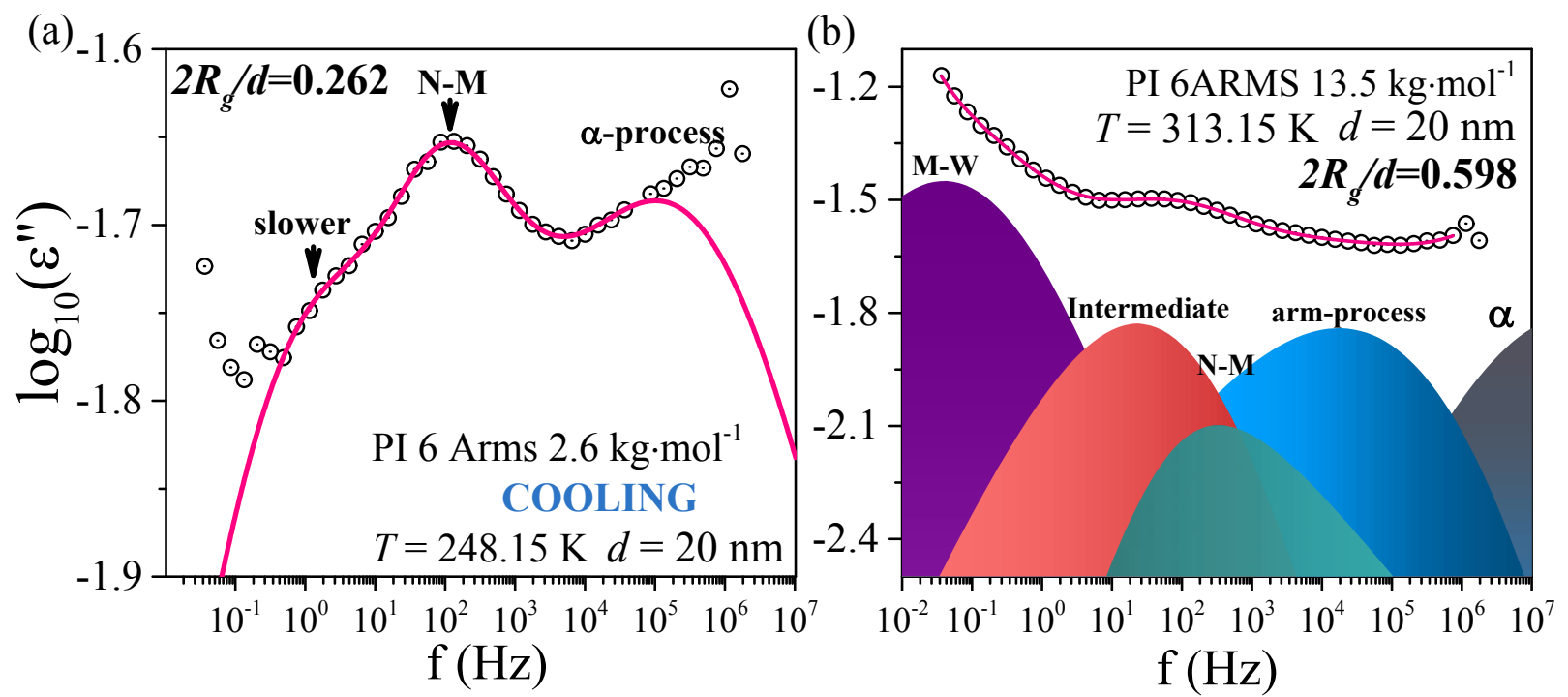

Figure S18. (a) Corrected (for the porosity) dielectric loss curve of star-shaped (a) SPI-6-2.6 and (b) SPI-6-13.5 under confinement in AAO templates with diameter of $20 \mathrm{~nm}$ (respective $2 R_{\mathrm{g}} / d=0.262$ and $2 R_{\mathrm{g}} / d=0.598$ ) obtained on cooling as function of frequency. The red line in (a) represents fit with a summation of three Havriliak-Negami functions corresponding to the segmental process, the $N M$ and the slower-process. The red line in (b) represents fit with a summation of five Havriliak-Negami functions for the segmental $\alpha$-process (gray), the arm-process (blue), the $N M$ (green), the intermediate (red) and the Maxwell-Wagner process (purple).

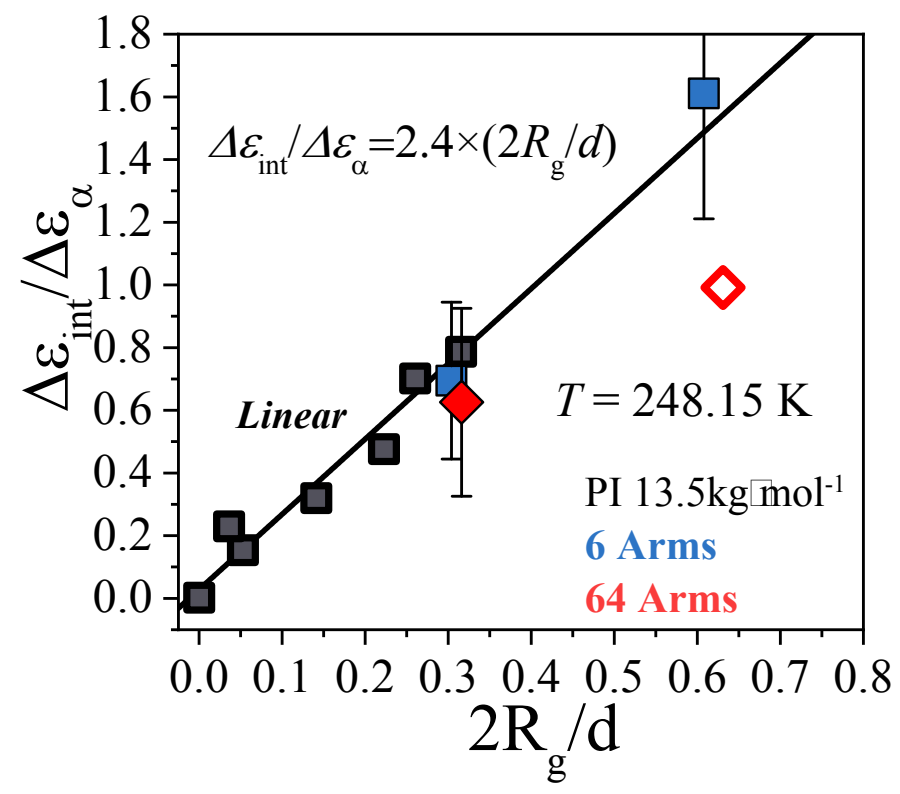

Figure S19. The dielectric strength of the intermediate process normalized by the corresponding strength for the segmental process for linear PI (black squares) ${ }^{6}$ and star-shaped PIs with: $f=6$ (blue squares), and $f=64$ (red rhombi) plotted as a function of the degree of confinement $2 R_{\mathrm{g}} / d$ at $T=$ $248.15 \mathrm{~K}$. The line is the result of a linear fit according to as $\Delta \varepsilon_{\text {inter }} / \Delta \varepsilon_{s} \sim 2.4 \times\left(2 R_{g} / d\right)$ from ref 6 . The empty symbol is an estimate star PI with $f=64$ located inside pores with a diameter of $20 \mathrm{~nm}$. 


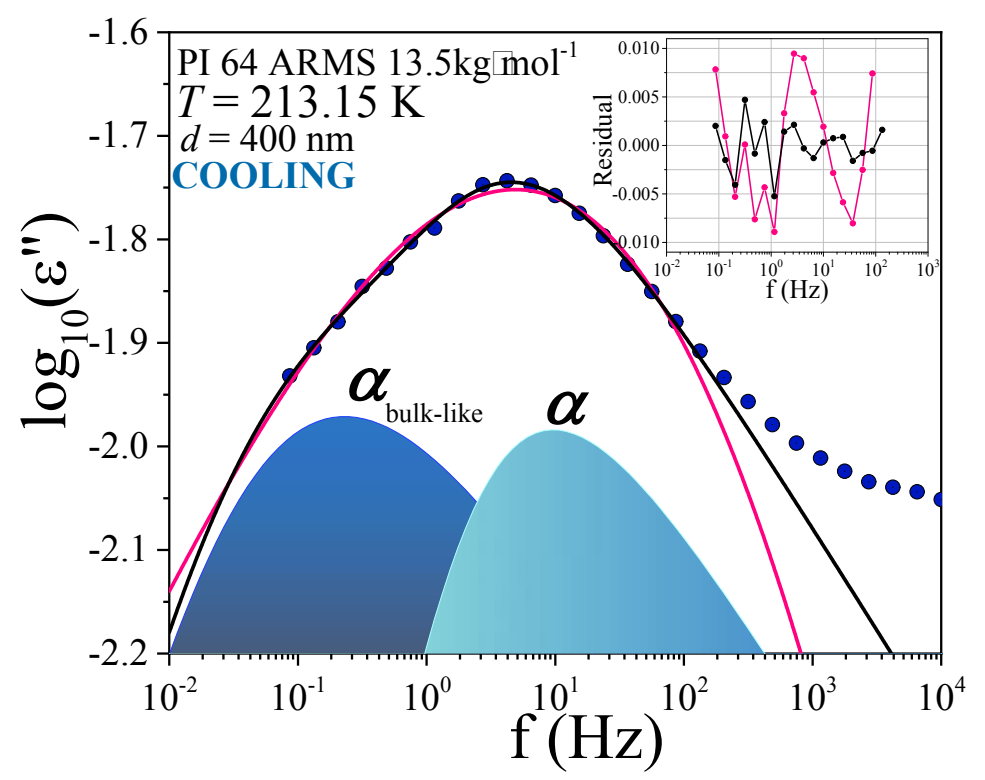

Figure S20. (a) Corrected dielectric loss curve of SPI-64-13.5 under confinement in AAO templates with diameter of $400 \mathrm{~nm}$ and $2 R_{\mathrm{g}} / d=0.030$ obtained on cooling at $T=213.15 \mathrm{~K}$. The pink line represents a fit with a single Havriliak-Negami function and the black line a fit with a summation of two $\mathrm{H}-\mathrm{N}$ functions corresponding to the segmental $\alpha_{\text {bulk-like-process (blue) and the faster } \alpha \text {-segmental }}$ process (light blue). In the inset, the residual plot is presented from the fit with a single and two $\mathrm{H}-\mathrm{N}$ functions is shown.

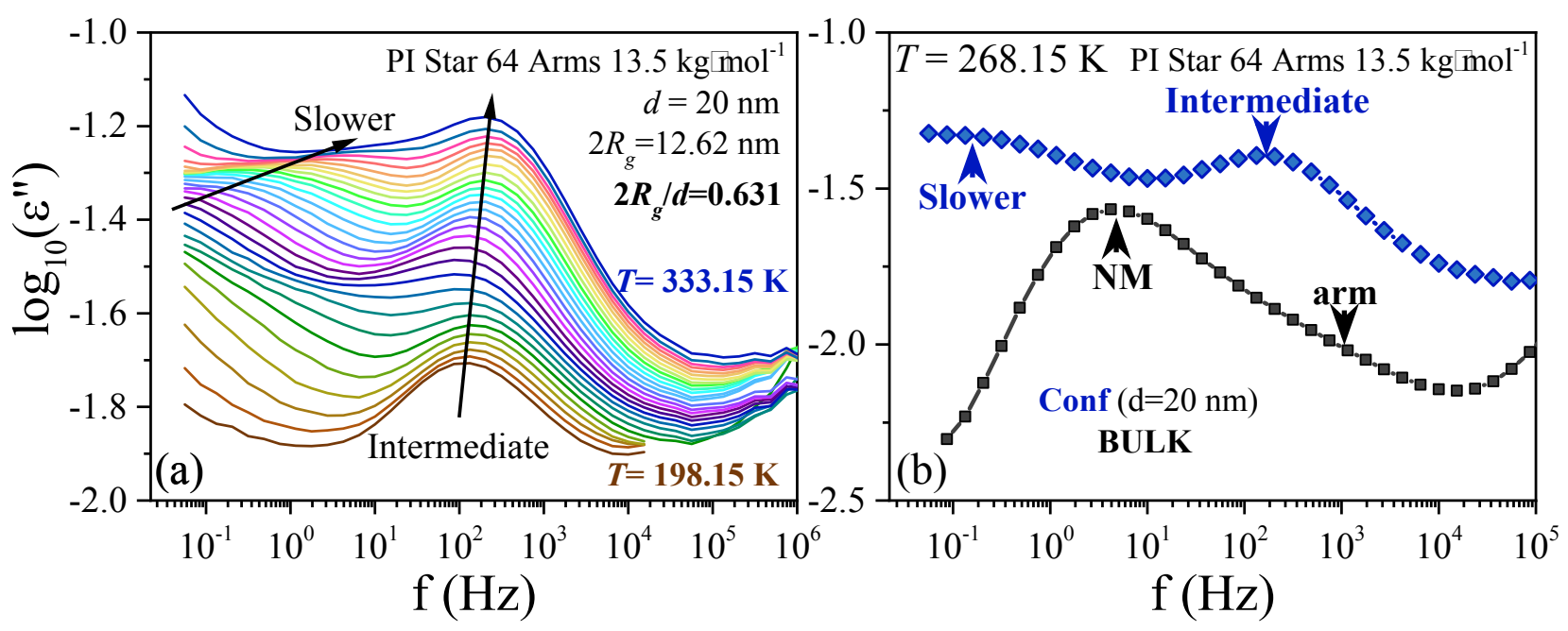

Figure S21. (a) The dielectric loss curves of SPI-64-13.5 under confinement inside self-ordered AAO with a pore diameter of $20 \mathrm{~nm}\left(2 R_{\mathrm{g}} / d=0.631\right)$. Data are shown within the temperature from $198.15 \mathrm{~K}$ to $333.15 \mathrm{~K}$. (b) Comparison of dielectric loss curve in bulk (black) and inside AAO with a pore diameter of $20 \mathrm{~nm}$ (blue) at $T=268.15 \mathrm{~K}$. Under confinement, two dominant processes are evident: the intermediate process and the slower (soft-colloidal) process. 


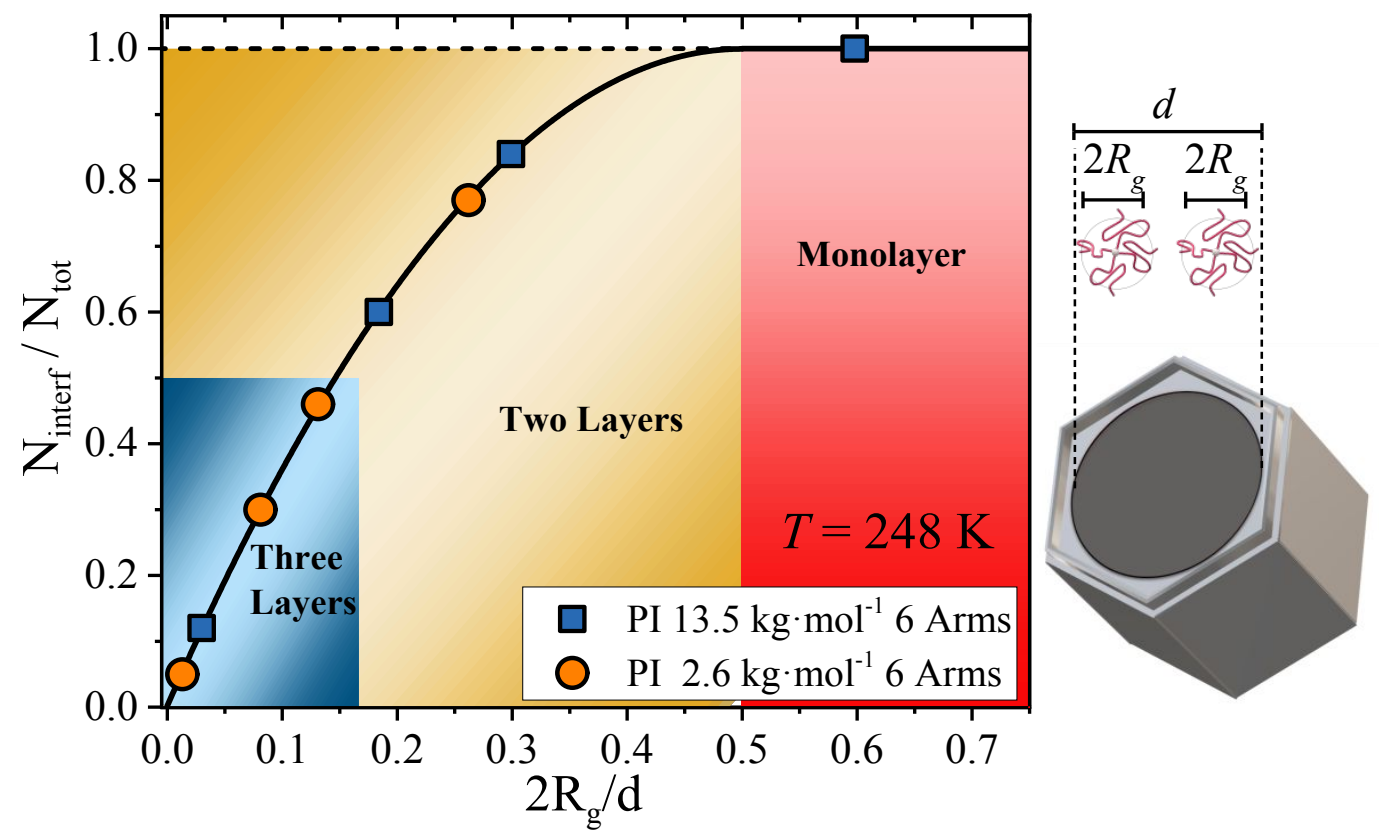

Figure S22. (a) Fraction of arms within the interfacial layer as a function of the degree of confinement $2 R_{\mathrm{g}} / d$ for the SPI-6-13.5 (blue) and SPI-6-2.6 (orange) at $T=248.15 \mathrm{~K}$. For $2 R_{\mathrm{g}} / d<0.5$ the number of arms, $N_{\text {interf }} / N_{\text {tot }}$, follows a parabolic dependence from a three-layer (blue) to a two-layer (yellow) regime and for $2 R_{\mathrm{g}}>0.5$ is equal to unit forming a single layer (monolayer, red).
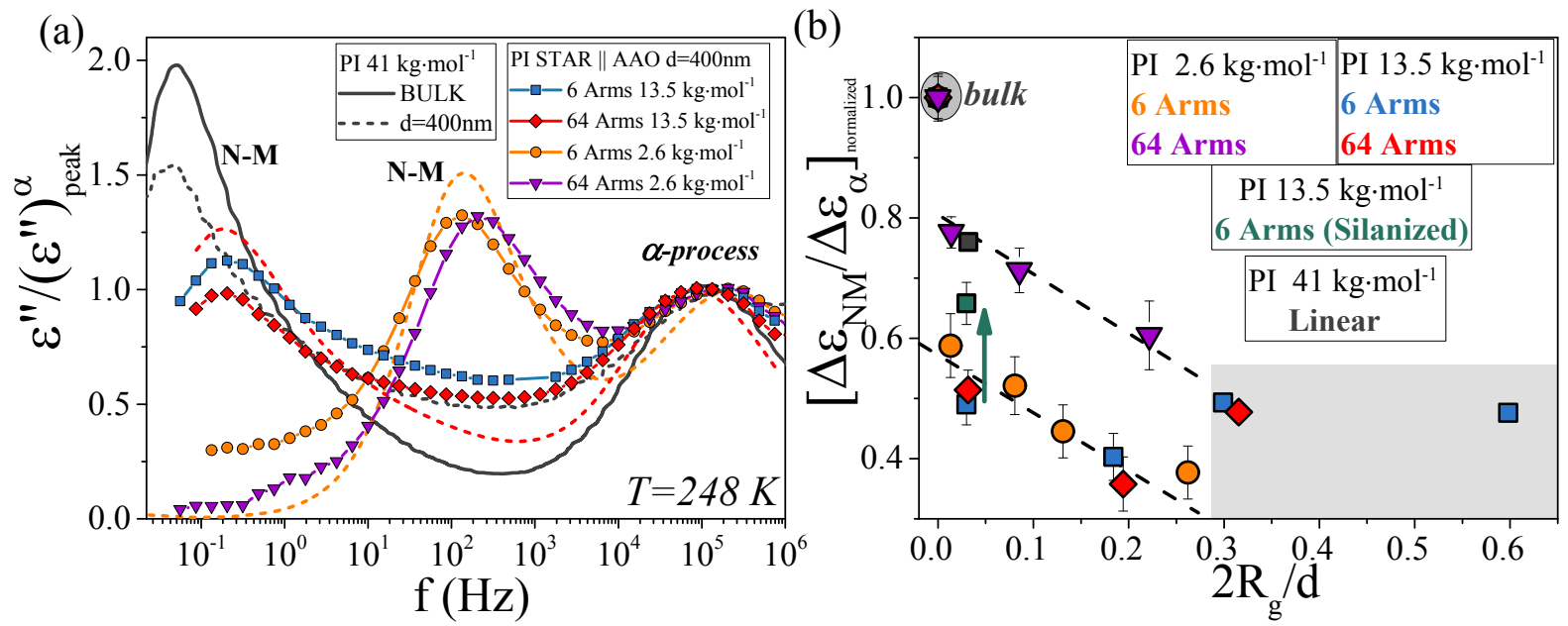

Figure S23. (a) Dielectric loss curves normalized to that of the segmental process for SPI-6-2.6 (orange), SPI-64-2.6 (purple), SPI-6-13.5 (blue) and SPI-64-13.5 (red) in comparison to linear PI with molecular mass of $42 \mathrm{~kg} \cdot \mathrm{mol}^{-1}$ under confinement in nanopores of $400 \mathrm{~nm}$ in diameter and in the bulk (dash line) at $T=248.15 \mathrm{~K}$. (b) Dielectric strength of the normal mode normalized by the corresponding dielectric strength of the segmental mode and further reduced to the value in the bulk for the linear PI (gray), the star PIs within the native and the treated (green) AAO. In the gray area the uncertainty is significantly higher. 
(1) Hadjichristidis, N.; Iatrou, H.; Pispas, S.; Pitsikalis, M. Anionic polymerization: High vacuum techniques. J. Polym. Sci., Part A: Polym. Chem. 2000, 38, 3211-3234.

(2) Uhrig, D.; Mays, J. W. Experimental techniques in high-vacuum anionic polymerization. $J$. Polym. Sci. A Polym. Chem. 2005, 43, 6179-6222.

(3) Roovers, J.; Zhou, L. L.; Toporowski, P. M.; van der Zwan, M.; Iatrou, H.; Hadjichristidis, N. Regular star polymers with 64 and 128 arms. Models for polymeric micelles. Macromolecules 1993, 26, 4324-4331.

(4) Pakula, T.; Vlassopoulos, D.; Fytas, G.; Roovers, J. Structure and dynamics of melts of multiarm polymer stars. Macromolecules 1998, 31, 8931-8940.

(5) Abou Elfadl; A., Kahlau; R., Herrmann; A., Novikov V. N.; Rossler, E. A. From rouse to fully established entanglement dynamics: a study of polyisoprene by dielectric spectroscopy. Macromolecules 2010, 43, 3340-3351.

(6) Politidis, C.; Alexandris, S.; Sakellariou, G.; Steinhart, M.; Floudas, G. Dynamics of entangled cis-1,4-polyisoprene confined to nanoporous alumina. Macromolecules 2019, 52, 4185-4195. 